This item was submitted to Loughborough's Research Repository by the author.

Items in Figshare are protected by copyright, with all rights reserved, unless otherwise indicated.

\title{
Large magnetoresistances and non-Ohmic conductivity in EuWO[1+x]N[2-x]
}

PLEASE CITE THE PUBLISHED VERSION

http://dx.doi.org/10.1063/1.3180813

\section{PUBLISHER}

(c) American Institute of Physics

\section{VERSION}

VoR (Version of Record)

\section{PUBLISHER STATEMENT}

This work is made available according to the conditions of the Creative Commons Attribution-NonCommercialNoDerivatives 4.0 International (CC BY-NC-ND 4.0) licence. Full details of this licence are available at: https://creativecommons.org/licenses/by-nc-nd/4.0/

\section{LICENCE}

CC BY-NC-ND 4.0

\section{REPOSITORY RECORD}

Kusmartseva, Anna F., Minghui Yang, Judith Oro-Sole, A.M. Bea, Alba Fuertes, and J. Paul Attfield. 2019. "Large Magnetoresistances and Non-ohmic Conductivity in Euwo[1+x]n[2-x]". figshare. https://hdl.handle.net/2134/22159. 


\section{A|P|P|lied Physics}

\section{Large magnetoresistances and non-Ohmic conductivity in EuWO $1+x$ N $2-x$}

A. Kusmartseva, M. Yang, J. Oró-Solé, A. M. Bea, A. Fuertes, and J. P. Attfield

Citation: Applied Physics Letters 95, 022110 (2009); doi: 10.1063/1.3180813

View online: http://dx.doi.org/10.1063/1.3180813

View Table of Contents: http://scitation.aip.org/content/aip/journal/apl/95/2?ver=pdfcov

Published by the AIP Publishing

\section{Articles you may be interested in}

Large anisotropic magnetoresistance of ruthenium-based Heusler alloys

J. Appl. Phys. 105, $07 E 513$ (2009); 10.1063/1.3080561

Large positive magnetoresistance effect below Curie temperature in In $1.90-x \mathrm{Mn} 0.1 \mathrm{Sn} \times \mathrm{O} 3$

J. Appl. Phys. 101, 09H121 (2007); 10.1063/1.2714192

Magnetic field-induced entropy change in phase-separated manganites

Appl. Phys. Lett. 89, 222503 (2006); 10.1063/1.2397535

Metal-insulator transition induced by $16 \mathrm{O}-18 \mathrm{O}$ oxygen isotope exchange in colossal negative magnetoresistance manganites

J. Appl. Phys. 83, 7369 (1998); 10.1063/1.367636

Influence of $\mathrm{Fe}$ in giant magnetoresistance ratio and magnetic properties of $\mathrm{La} 0.7 \mathrm{Ca} 0.3 \mathrm{Mn} 1-\mathrm{x} \mathrm{Fe} \times \mathrm{O} 3$ perovskite type compounds

J. Appl. Phys. 81, 5767 (1997); 10.1063/1.364721

\section{Precise temperature control for cryogenic research}

\section{Model 372}

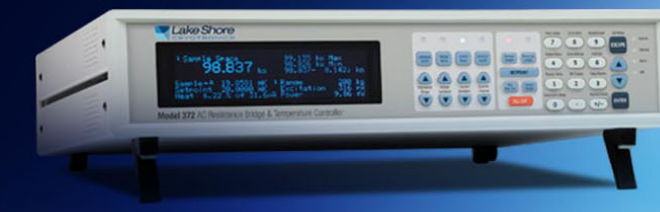




\title{
Large magnetoresistances and non-Ohmic conductivity in EuWO ${ }_{1+x} \mathbf{N}_{2-x}$
}

\author{
A. Kusmartseva, ${ }^{1}$ M. Yang, ${ }^{1}$ J. Oró-Solé, ${ }^{2}$ A. M. Bea, ${ }^{2}$ A. Fuertes,${ }^{2}$ and J. P. Attfield ${ }^{1, a)}$ \\ ${ }^{1}$ Centre for Science at Extreme Conditions and School of Chemistry, University of Edinburgh, \\ Mayfield Road, Edinburgh EH9 3JZ, United Kingdom \\ ${ }^{2}$ Institut de Ciència de Materials de Barcelona (C.S.I.C.), Campus U.A.B., 08193 Bellaterra, Spain
}

(Received 13 May 2009; accepted 26 June 2009; published online 16 July 2009)

\begin{abstract}
The magnetic field and voltage dependent electronic transport properties of $\mathrm{EuWO}_{1+x} \mathrm{~N}_{2-x}$ ceramics are reported. Large negative magnetoresistances are observed at low temperatures, up to $70 \%$ in the least doped $(x=0.09)$ material. Non-Ohmic conduction emerges below the $12 \mathrm{~K}$ Curie transition. This is attributed to a microstructure of ferromagnetic conducting and antiferromagnetic insulating regions resulting from small spatial fluctuations in the chemical doping. () 2009 American Institute of Physics. [DOI: 10.1063/1.3180813]
\end{abstract}

Materials in which the electrical current is coupled to magnetism or shows nonlinear variation with voltage are of fundamental interest and practical importance for technologies such as magnetoresistive random access memory ${ }^{1}$ and resistive random access memory. ${ }^{2}$ Many oxide families are known to have such properties, but the utility of an unexplored class of materials-europium(II) oxynitride perovskites-was recently demonstrated as colossal negative magnetoresistances (MRs) were discovered at low temperatures. ${ }^{3}$ The presence of nitride enables $d^{0}$ transition metals in high formal oxidation states to be stabilized, e.g., $\mathrm{Nb},{ }^{3} \mathrm{Ta},{ }^{3,4}$ and $\mathrm{W} .{ }^{5}$ Nitrogen-deficiency leads to electrondoping of the transition metal $d$-bands and the carrier transport may be strongly coupled to the magnetization from the $\mathrm{Eu}^{2+} S=7 / 2$ core spins, leading to $-\mathrm{MR}>99 \%$ for $\mathrm{EuNbO}_{2+x} \mathrm{~N}_{1-x}(\mathrm{MR}=[\rho(H)-\rho(0)] / \rho(0) ; \rho$ is resistivity, measured in zero field and magnetic field $H$ ). Here we report the magnetotransport properties of the correspondingly electron-doped material EuWO ${ }_{1+x} \mathrm{~N}_{2-x}$, in which large negative MRs and microstructurally induced nonohmicity are discovered.

EuWO ${ }_{1+x} \mathrm{~N}_{2-x}$ samples with $x=0.09,0.17$, and 0.25 were prepared by ammonolysis of a precursor $\mathrm{Eu}_{2} \mathrm{~W}_{2} \mathrm{O}_{9}$, synthesized by heating $\mathrm{Eu}_{2} \mathrm{O}_{3}$ and $\mathrm{WO}_{3}$ in air at $1100{ }^{\circ} \mathrm{C}$ for $72 \mathrm{~h}$ with intermediate regrinding. $\mathrm{Eu}_{2} \mathrm{~W}_{2} \mathrm{O}_{9}$ powder was nitrided at $800{ }^{\circ} \mathrm{C}$ during $15 \mathrm{~h}$ in flowing $99.9 \% \mathrm{NH}_{3}$ and quenched to room temperature under ammonia. $\mathrm{N}$ contents were controlled by varying the flow rate between 200 and $270 \mathrm{~cm}^{3} / \mathrm{min} ; x$ decreases as the flow rate increases. $7 \mathrm{~mm}$ diameter pellets of the EuWO ${ }_{1+x} \mathrm{~N}_{2-x}$ products for electrical measurements were pressed at 3 tons and subjected to a second ammonia treatment for $3 \mathrm{~h}$ under the same conditions. $\mathrm{N}$ contents were determined by combustion analysis.

The EuWO ${ }_{1+x} \mathrm{~N}_{2-x}$ samples are single phase perovskites and appear cubic by powder x-ray diffraction [Fig. 1(a)] but electron diffraction revealed a body-centered $\sqrt{ } 2 a_{p} \times \sqrt{ } 2 a_{p}$ $\times 2 a_{p}$ supercell as was also observed in $\mathrm{EuNbO}_{2} \mathrm{~N}$. ${ }^{3}$ The cubic lattice parameter $a_{p}$ increases slightly with the nitrogen deficiency $x\left(a_{p}=3.9592(4), 3.9622(2)\right.$ and 3.9675(2) $\AA$ for $x=0.09,0.17$, and 0.25 , respectively) due to lattice expansion from electron doping into the $\mathrm{W}: 5 \mathrm{~d}$ band (reduction of $\mathrm{W}^{6+}$ to $\mathrm{W}^{5+}$ ). The presence of $\mathrm{Eu}^{2+}$, as found in the $\mathrm{EuMO}_{2} \mathrm{~N}$

${ }^{a)}$ Electronic mail: j.p.attfield@ed.ac.uk.
$(M=\mathrm{Nb}, \mathrm{Ta})$ analogs,${ }^{3}$ is confirmed by the mean Eu-O/N distances of 2.80-2.81 $\AA$ in these pseudocubic structures (cf. 2.88 and $2.68 \AA$, respectively, predicted from ionic radii of $\mathrm{Eu}^{2+}$ and $\mathrm{Eu}^{3+}$ ) but full determinations of the superstructure will be needed to obtain accurate distances. Magnetization measurements show that the three samples order ferromagnetically at $12 \mathrm{~K}$, as observed in a previous study of an $x=0.58$ sample, ${ }^{5}$ with no variation in $T_{C}$ to within $\pm 0.5 \mathrm{~K}$. Magnetization $(M)$ - field $(H)$ loops [Fig. 1(b)] confirm the ferromagnetic order although the saturated moment of $4.1 \mu_{B}$ is significantly reduced from the theoretical value of $7 \mu_{B}$ for $\mathrm{Eu}^{2+}$, suggesting that spin canting or coexisting ferromagnetic and antiferromagnetic phases may be present, as discussed later.

The electronic transport properties of the three EuWO $_{1+x} \mathrm{~N}_{2-x}$ samples were measured using a Quantum Design physical properties measurement system down to $2 \mathrm{~K}$ and in fields up to $7 \mathrm{~T}$, and a closed-cycle refrigerator operating down to $11 \mathrm{~K}$. All resistivity measurements were carried out in the conventional four-point setup, where an ac current $(I)$ of $0.5 \mathrm{~mA}$ was applied and the voltage $(V)$ drop across the samples was registered. The resistivity of $\mathrm{EuWO}_{1+x} \mathrm{~N}_{2-x}$ decreases with the electron-doping $x$ and the temperature variations in Fig. 2(a) are typical for heavily



FIG. 1. (Color online) (a) Fit of a cubic perovskite model to the x-ray diffraction pattern and (b) magnetization-field loops, for $\mathrm{EuWO}_{1+x} \mathrm{~N}_{2-x}$ $(x=0.17$ sample). 
(a)

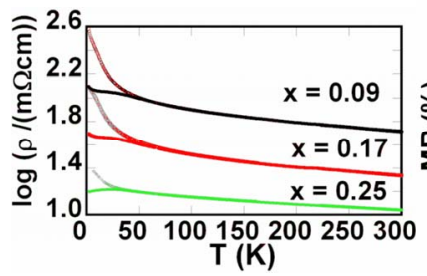

(b)



(c)

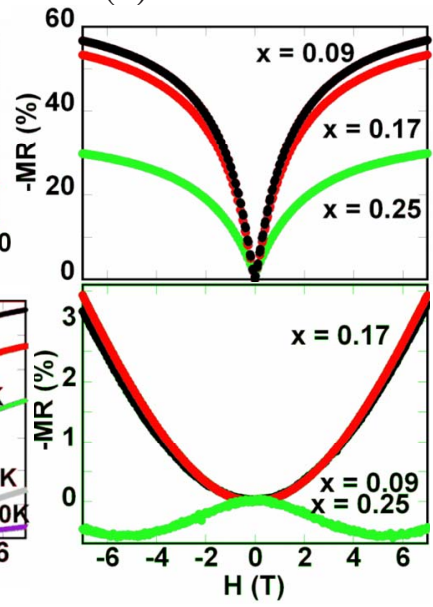

FIG. 2. (Color online) MR data for $\mathrm{EuWO}_{1+x} \mathrm{~N}_{2-x}$ ceramics; (a) resistivities $\rho$ for three samples in zero field (upper branch at low temperatures) and in a magnetic field of $7 \mathrm{~T}$ (lower branch at low temperatures), (b) - MR vs field at temperatures of $2-50 \mathrm{~K}$ for the $x=0.09$ sample, (c) - MR vs field for the three samples at $8 \mathrm{~K}$ (upper panel) and $50 \mathrm{~K}$ (lower panel).

doped semiconductors. Giant negative MRs are evidenced by the divergence of zero-field and in-field resistivities below $\sim 50 \mathrm{~K}$ for $x=0.09$ and 0.17 and $\sim 30 \mathrm{~K}$ for $x=0.25$ samples. This corresponds to the onset of ferromagnetic fluctuations as evidenced by the nonlinearity of $M-H$ below $50 \mathrm{~K}$ observed in Fig. 1(b) for $x=0.17$.

The field variation for the least doped $(x=0.09)$ sample is shown in Fig. 2(b) - this displays the highest $-\mathrm{MR} \approx 70 \%$ at $2 \mathrm{~K}$ and $7 \mathrm{~T}$. The thermal evolution of $-\mathrm{MR}(H)$ follows an approximate $-\mathrm{MR} \sim M^{2}$ relationship typical for lightly doped ferromagnetic insulators, so that $-\mathrm{MR}$ changes from an $H^{2}$ dependence at $50 \mathrm{~K}$ in the paramagnetic region, to a sharp low field increase below $T_{C}$. Comparison of the MRs of the three samples in the ferromagnetic region [Fig. 2(c)] shows a decrease in the magnitude of -MR with $x$ as electron hopping becomes less coupled to the magnetization in the more conducting materials. The $50 \mathrm{~K}-\mathrm{MR}(H)$ curves in Fig. 2(c) show a different behavior between the $x=0.09$ and $x=0.17$ samples, which show virtually identical $\sim H^{2}$ dependences driven by ferromagnetic fluctuations, and the $x$ $=0.25$ material in which the fluctuations are less pronounced and a small positive MR effect typical of paramagnetic semiconductors is observed.

During the magnetotransport measurements it became apparent that the three EuWO ${ }_{1+x} \mathrm{~N}_{2-x}$ ceramics showed nonOhmic conductivity at low temperatures and voltages. Systematic de $I-V$ studies as a function of temperature were carried out in the $0-5 \mathrm{~mA}, 0-500 \mu \mathrm{V}$ range to explore this nonohmicity. The effects are most pronounced for the $x=0.25$ sample, for which the $I-V$ isotherms are shown in Fig. 3. The $I-V$ behavior evolves from a linear, Ohmic relationship at high temperatures to the curve at $3 \mathrm{~K}$, which changes from a Schottky (rectification) behavior at low $V$ to an Ohmic variation at higher voltages. To explore the crossover we have fitted the isotherms down to $4 \mathrm{~K}$ as $I=A V^{n}$ $+B V$, by varying the coefficients $A$ and $B$ and the exponent $n$. The temperature variation of $n$ (Fig. 3 inset) captures the emergence of the Schottky term; the divergence of $n$ above the ohmic $n=1$ limit coincides with the $12 \mathrm{~K}$ Curie transition. Hence, the ferromagnetic order drives the electrical change from ohmic to a nonlinear conducting regime in

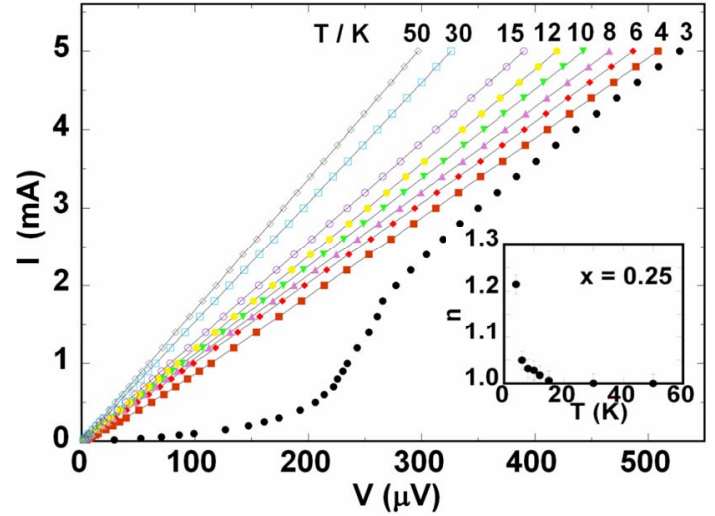

FIG. 3. (Color online) Current-voltage isotherms for the $x=0.25$ sample of EuWO $_{1+x} \mathrm{~N}_{2-x}$ at temperatures above (open symbols) and below (closed symbols) the $12 \mathrm{~K}$ Curie transition. Inset shows the thermal behavior of the exponent $n$ from fitting the equation $I=A V^{n}+B V$ to isotherms at $4 \mathrm{~K}$ and above.

which both Schottky and Ohmic contributions are significant.

Non-Ohmic conduction arises from the presence of electrical inhomogeneities such as metallic and insulating regions which may be modeled as an appropriate arrangement of resistors and capacitors. For example, a composite of silver-coated glass spheres showed an $I=A V^{n}$ variation with $1<n<2,{ }^{6}$ and other models have been suggested for metal/ semiconductor composite networks. ${ }^{7-9}$ Nonlinear conductivity was also observed in charge ordered manganite perovskites, and may be linked to the melting of charge order and creation of conducting filaments above a certain threshold current. $^{10-12}$ This is qualitatively different to our findings where the nonlinear conduction occurs predominantly at low currents and appears to be intrinsically linked to the ferromagnetic ordering.

A likely explanation of the non-Ohmic behavior is that some regions within the EuWO ${ }_{1+x} \mathrm{~N}_{2-x}$ samples are metallized below $T_{C}$. The effect is most pronounced in the most highly doped, $x=0.25$ sample, and accompanies a downturn (bulk metallic percolation) in the high field resistivity curve [Fig. 2(a)]. Small electrical inhomogeneities can arise from a slightly inhomogenous $\mathrm{O} / \mathrm{N}$ (doping) distribution created during the sintering of the ceramic pellets, although this does not usually give rise to such pronounced nonohmicity. We propose that the effect of this slight inhomogeneity is amplified by the low temperature magnetic order in $\mathrm{EuWO}_{1+x} \mathrm{~N}_{2-x}$. The saturated moment of $4.1 \mu_{B}$ [Fig. 1(b)] has only $60 \%$ of the expected value, showing that substantial canting or segregation into ferro- and antiferromagnetic phases occurs. The latter picture is supported by the properties of the related material $\mathrm{EuTiO}_{3}$, which is antiferromagnetic in zero field but shows a field-induced ferromagnetism. ${ }^{13}$ Small compositional fluctuations in $\mathrm{EuWO}_{1+x} \mathrm{~N}_{2-x}$ lead to spatial variations in the ferromagnetic order parameter, resulting a microstructure of ferromagnetic metallic and insulating antiferromagnetic regions that conducts nonohmically. This microstructure is also sensitive to the electric field; high voltages further metallize the sample and Ohmic behavior is recovered, as observed in the $3 \mathrm{~K}$ isotherm on Fig. 3 .

To conclude, EuWO ${ }_{1+x} \mathrm{~N}_{2-x}$ ceramics displays large negative MRs and magnetically driven nonohmicity at low temperatures. The MR results from coupling of the doped W 
d-band carriers to the $\mathrm{Eu}^{2+}$ magnetization, and so is sensitive to the doping, field and temperature. Non-Ohmic behavior emerges from spatial fluctuations in the ferromagnetic order that create a microstructure of insulating and conducting regions. Manipulation of the $\mathrm{O} / \mathrm{N}$ content within $\mathrm{EuWO}_{1+x} \mathrm{~N}_{2-x}$ films or monoliths could thus be used to create spintronic or nonlinear resistive devices operating at low temperatures.

This work was supported by the Ministerio de Ciencia e Innovación (Grant Nos. MAT2008-04587 and PR2008-0164) and the Generalitat de Catalunya, Spain, and the Royal Society, EPSRC, and the Leverhulme Trust, U.K.

${ }^{1}$ S. Parkin, X. Jiang, C. Kaiser, A. Panchula, K. Roche, and M. Samant, Proc. IEEE 91, 661 (2003).

${ }^{2}$ R. Waser and M. Aono, Nature Mater. 6, 833 (2007).
${ }^{3}$ A. B. Jorge, J. Oró-Solé, A. M. Bea, N. Mufti, T. T. Palstra, J. A. Rodgers, J. P. Attfield, and A. Fuertes, J. Am. Chem. Soc. 130, 12572 (2008).

${ }^{4}$ R. Marchand, F. Pors, and Y. Laurent, Ann. Chim. - Sci. Mat. 16, 553 (1991).

${ }^{5}$ R. Pastrana-Fabregas, J. Isasi-Marin, C. Cascales, and R. Sáez-Puche, J. Solid State Chem. 180, 92 (2007).

${ }^{6}$ H. Sodolski, R. Zielinski, T. Slupkowski, and B. Jachym, Phys. Status Solidi A 32, 603 (1975).

${ }^{7}$ S. H. Kwan, F. G. Shin, and W. L. Tsui, J. Mater. Sci. 15, 2978 (1980).

${ }^{8}$ T. J. Coutts, Thin Solid Films 38, 313 (1976).

${ }^{9}$ S. M. Aharoni, J. Appl. Phys. 43, 2463 (1972).

${ }^{10}$ Y. Tokura and N. Nagaosa, Science 288, 462 (2000).

${ }^{11}$ A. Guha, A. K. Raychaudhuri, A. R. Raju, and C. N. R. Rao, Phys. Rev. B 62, 5320 (2000).

${ }^{12}$ S. Mercone, A. Wahl, Ch. Simon, and C. Martin, Phys. Rev. B 65, 214428 (2002).

${ }^{13}$ T. Katsufuji and H. Takagi, Phys. Rev. B 64, 054415 (2001). 